Pacific Journal of Mathematic 


\title{
HOMEOMORPHIC MEASURES IN THE HILBERT CUBE
}

\author{
John C. Oxtoby and Vidhu S. PRAsad
}

\begin{abstract}
A Borel probability measure $\mu$ in the Hilbert cube is homeomorphic to the usual product measure if and only if it is positive for nonempty open sets and zero for points. The transformation can be effected by a homeomorphism equal to the identity on any prescribed $\mu$-null $Z$-set. Several extension, approximation, and embedding theorems are obtained as applications.
\end{abstract}

1. Introduction and basic theorem. Let $Q=I^{\infty}=\Pi_{1}^{\infty} I_{i}$ denote the Hilbert cube, where each $I_{i}=[0,1]$, and let $\lambda$ denote Lebesgue product measure in $Q$ restricted to Borel sets. - If $h$ is any homeomorphism of $Q$ onto itself, then $E \mapsto \lambda(h(E))$ defines a Borel measure in $Q$ which we shall denote by $\lambda h$. Any measure $\mu$ that admits such a representation is said to be homeomorphic (or topologically equivalent) to $\lambda$. Evidently any such measure in $Q$ is a normalized Borel measure and it must be zero for points (nonatomic) and positive for nonempty open sets (locally positive). Our aim is to prove the converse of this statement, with appropriate refinements specifying what kinds of subsets can be kept fixed, and to give several applications of this result: an extension theorem for measure preserving homeomorphisms between subsets of $Q$, a version of Luzin's theorem for measure preserving homeomorphisms, and a characterization of the topological measure spaces that can be embedded in $Q$ by a measure preserving homeomorphism. The potential usefulness of such a theorem in connection with the last mentioned problem has long been recognized, especially by Dorothy Maharam and A. H. Stone (cf. [9] and [10]). We include also a couple of related results which do not depend on the basic theorem: a characterization of sets topologically equivalent to nullsets, and a version of Goffman's approximation theorem for one-one measurable transformations, both of which were previously known only in the finite-dimensional case.

We wish to thank Steve Alpern for many helpful discussions.

It is known [7] (see also [4]) that to characterize measures homeomorphic to Lebesgue measure in $I^{n}(n$ finite) it is necessary to require that $\mu\left(\partial I^{n}\right)=0$ in addition to the requirements mentioned above. Since $I^{\infty}$ has no boundary it is not surprising that this condition can be dropped but it is remarkable that no other condition is needed to replace it.

Let $Q$ be given the metric $d(x, y)=\sum_{1}^{\infty}\left|x_{i}-y_{i}\right| / 2^{i}$. By an $r$-set 
we shall mean a subset of $Q$ of the form $R=\Pi_{1}^{\infty}\left[a_{i}, b_{i}\right]$ where $0 \leqq$ $a_{i}<b_{i} \leqq 1$ for all $i$, and $\left[a_{i}, b_{i}\right]=I_{i}$ for all but finitely many values of $i$; that is, the closure of a basic open set. A set of the form $\left\{x \in R: x_{i}=c\right\}$ with $a_{i} \leqq c \leqq b_{i}$ is called a section or a face of $R$ according as $a_{i}<c<b_{i}$ or not. The union of all the faces of $R$ is a dense subset of $R$ called its pseudoboundary; it will be denoted by $\delta R$. The topological boundary $\partial R$ of $R$ is the union of the (finitely many) faces of $R$ for which $a_{i}>0$ or $b_{i}<1$. The set $s=$ $Q-\delta Q$ is called the pseudointerior of $Q$.

Let $H(Q)$ denote the space of all homeomorphisms of $Q$ onto itself with the topology of uniform convergence and the norm $\|f\|=$ $\sup _{x \in Q} d(x, f(x)) . H(Q)$ is a complete metric group with respect to the metric $\rho(f, g)=\max \left\{\left\|f g^{-1}\right\|,\left\|f^{-1} g\right\|\right\}$. Let

$$
H_{0}(Q)=\{h \in H(Q): h(W)=W \text { for each face } W \text { of } Q\} \text {. }
$$

When $B \subset Q$ and $h(x)=x$ for all $x \in B, h$ is said to leave $B$ fixed. Let

$$
H(Q, B)=\{h \in H(Q): h \text { leaves } B \text { fixed }\}
$$

and

$$
H_{0}(Q, B)=\left\{h \in H_{0}(Q): h \text { leaves } B \text { fixed }\right\} .
$$

Each of these is a closed subgroup of $H(Q)$.

The following lemma, which has no analogue for $I^{n}$, plays an important role; it serves to reduce the general problem to the case of measures that vanish on $\delta Q$. Methods similar to those used in the case of $I^{n}$ can then be applied.

LeMma 1. If $\mu$ is a finite Borel measure in $Q$ and $B$ is the union of a finite number of faces of $Q$ with $\mu(B)=0$, then $\mu h(\delta Q)=$ 0 for all $h$ in a certain dense $G_{o}$ subset of $H(Q, B)$.

Proof. Let $W=\left\{x \in Q: x_{k}=c\right\}, c=0$ or 1 , be an arbitrary face of $Q$ that is not contained in $B$. For each positive integer $j$ let

$$
E_{j}=\{h \in H(Q, B): \mu h(W)<1 / j\} .
$$

It is clear that $E_{j}$ is an open subset of $H(Q, B)$. For any $j$ and $0<\varepsilon<1$ choose an integer $n \neq k$ with $1 / 2^{n}<\varepsilon / 2$ such that neither of the faces $x_{n}=0$ or 1 is contained in $B$. Let $\delta<\varepsilon / 2^{k+1}$ be a positive number such that the $\delta$-neighborhood $B_{\delta}$ of $B$ satisfies $\mu\left(B_{i}\right)<1 / 2 j$. Choose an interval $(a, b) \subset I_{n}$ such that

$$
\mu\left(\left\{x \in Q: a<x_{n}<b\right\}\right)<1 / 2 j
$$


and define a continuous family $\bar{h}_{t}(0 \leqq t \leqq 1)$ of homeomorphisms of the square $I_{k} \times I_{n}$ onto itself such that $(i) \bar{h}_{t}$ leaves fixed all points of $I_{k} \times I_{n}$ for which $\left|x_{k}-c\right| \geqq \delta$, (ii) $\bar{h}_{0}$ is the identity, and (iii) $\bar{h}_{1}$ contracts the edge $\{c\} \times I_{n}$ to a subset of $\{c\} \times(a, b)$. Let $F$ be the union of the faces of $Q$ that are contained in $B$ and meet $W$. For each $x \in Q$ let $s(x)=\min \{d(x, F) / \delta, 1\}$ and define $h(x)=\left(x_{i}^{\prime}\right)$ by setting $\left(x_{k}^{\prime}, x_{n}^{\prime}\right)=\bar{h}_{s(x)}\left(x_{k}, x_{n}\right)$ and $x_{i}^{\prime}=x_{i}$ for all $i \neq k, n$. Then $h \in H(Q$, $B)$. Each section of $Q$ of the form $x_{i}=$ constant, $i \neq k, n$, is invariant under $h$, and the $\delta$-neighborhood of the face of $Q$ opposite $W$ is fixed, therefore $h\left(B_{\hat{o}}\right) \subset B_{j}$. Hence

$$
h(W)=h\left(W \cap B_{\dot{o}}\right) \cup h\left(W-B_{\delta}\right) \subset B_{\delta} \cup\left\{x \in Q: a<x_{n}<b\right\} .
$$

Therefore $\mu h(W)<1 / 2 j+1 / 2 j$ and so $h \in E_{j}$. Since $\|h\| \leqq \delta / 2^{k}+$ $1 / 2^{n}<\varepsilon$ this shows that $E_{j}$ contains elements with arbitrarily small norm.

For any $g \in H(Q, B)$ let $\nu=\mu g$. Then $\nu(B)=0$, and as just shown there exists an $h \in H(Q, B)$ with arbitrarily small norm such that $\nu h(W)<1 / j$. Then $g h$ is an element of $E_{j}$ arbitrarily near $g$. Thus $E_{j}$ is a dense open subset of $H(Q, B)$. Intersecting over $j$ and the countably many faces of $Q$ that are not contained in $B$ completes the proof.

The following basic theorem extends to $I^{\infty}$ the result embodied in Theorem 2 of [7].

THEOREM 1. Let $\mu$ be a nonatomic, locally positive, normalized Borel measure in $Q$, and let $B$ be the union of a finite number of faces of $Q$ with $\mu(B)=0$. Then $\mu=\lambda f$ for some $f \in H(Q, B)$. If $\mu(\delta Q)=0$ then $\mu=\lambda f$ for some $f \in H_{0}(Q, B)$.

By virtue of Lemma 1 it is sufficient to prove the last statement. Note that in Lemma 1 and Theorem 1 the measure $\mu$ may be confined to $\delta Q$ and the set $B$ may necessarily be empty; for example, $\mu$ may be a normalized sum of product measures on the faces of $Q$. First we prove an easy lemma.

Lemma 2. Let $\mu$ be a finite nonatomic Borel measure in $Q$, let $0 \leqq \alpha<\beta \leqq \mu(Q)$ be given, and let $F$ be a closed subset of $Q$ with $\mu(F)=0$. There exists an open set $G$ such that $G \cap F=\varnothing$ and $\alpha<\mu(G)<\beta$.

Proof. Since $Q$ is compact and points have measure zero there is a number $\eta>0$ such that $\mu(U)<\beta-\alpha$ for every open set $U$ with diameter less than $\eta$. Choose a compact subset $K$ of $Q-F$ 
with $\mu(K)>\alpha$ and let $\delta$ be a positive number less than $\eta$ such that $Q-F$ contains the $\delta$-neighborhood of $K$. Divide $Q$ into $r$-sets of diameter less than $\delta$ using sections that have $\mu$-measure zero. Let $\left\{U_{1}, \cdots, U_{n}\right\}$ be the interiors of the members of this partition that meet $K$ and let $G=U_{1} \cup \cdots \cup U_{k}$, where $k$ is the least integer such that $\mu(G)>\alpha$. Then $\alpha<\mu(G)<\beta$ and $G \cap F=\varnothing$.

LEMma 3 (cf. Lemma 7 of [7]). Let $\mu$ be a finite nonatomic Borel measure in $Q$ with $\mu(\delta Q)=0$, let $B$ be the union of a finite number of faces of $Q$, and let $R_{1}=\left\{x \in Q: x_{k} \leqq c\right\}$ and $R_{2}=\left\{x \in Q: x_{k} \geqq\right.$ c) be the two r-sets into which $Q$ is divided by the section $P=\{x \in$ $\left.Q: x_{k}=c\right\}$. Then for any two positive numbers $\alpha_{1}$ and $\alpha_{2}$ with $\alpha_{1}+$ $\alpha_{2}=\mu(Q)$ there is an $h \in H_{0}(Q, B)$ such that $\mu h\left(R_{i}\right)=\alpha_{i}(i=1,2)$.

Proof. Let $H$ denote the set of all $h \in H_{0}(Q, B)$ such that $\mu h\left(R_{i}\right) \geqq \alpha_{i}(i=1,2) . \quad H$ is a closed subset of $H_{0}(Q, B)$. To show that $H$ is nonempty note that unless it contains the identity we must have either $\mu\left(R_{1}\right)<\alpha_{1}$ or $\mu\left(R_{2}\right)<\alpha_{2}$. Assume that $\mu\left(R_{1}\right)<\alpha_{1}$ and define $h_{t}: Q \rightarrow Q$ for $0<t<\infty$ as follows. Let $F$ denote the union of the faces of $Q$ that are contained in $B$ and meet $P$. For $x \in Q$ let $s(x)=\min \{d(x, F), 1 / 2\}$ and define $h_{t}(x)=\left(x_{i}^{\prime}\right)$ by setting $x_{i}^{\prime}=x_{i}$ for all $i \neq k$ and letting $x_{k}^{\prime}$ be the piecewise linear function of $x_{k}$ that maps $0, c, 1$ into $0, c+(1-c) s(x)^{1 / t}, 1$, respectively. Then $h_{t} \in H_{0}(Q, B) . \mu\left(h_{t}\left(R_{1}\right)\right)$ is monotone increasing and continuous on the right. It tends to $\mu\left(R_{1}\right)$ as $t \rightarrow 0$ and to $\mu(Q)$ as $t \rightarrow \infty$, and $\mu\left(R_{1}\right)<$ $\alpha_{1}<\mu(Q)$. Hence there is a least number $\tau$ such that $\mu h_{\tau}\left(R_{1}\right) \geqq \alpha_{1}$. Since $\mu\left(h_{t}\left(R_{2}\right)\right)$ is monotone decreasing, continuous on the left, and greater than $\alpha_{2}$ for $0<t<\tau$, it follows that $\mu h_{\tau}\left(R_{2}\right) \geqq \alpha_{2}$. Thus $h_{\tau} \in H$. Similar reasoning shows that $H$ is nonempty in case $\mu\left(R_{2}\right)<$ $\alpha_{2}$.

For each positive integer $n$ let

$$
H_{n}=\left\{h \in H: \mu h\left(R_{1}\right) \geqq \alpha_{1}+\frac{1}{n}\right\} .
$$

Then $H_{n}$ is a closed subset of $H$. To show that $H_{n}$ is nowhere dense relative to $H$ let $g \in H_{n}$ and define $\nu=\mu g$. Then $\nu$ is a finite nonatomic Borel measure in $Q$ with $\nu(\delta Q)=0$ and $\nu(P) \geqq 1 / n$. Putting $\alpha=\nu\left(R_{1}\right)-\alpha_{1}-1 / n$ and $\beta=\nu\left(R_{1}\right)-\alpha_{1}$ we have $0 \leqq \alpha<\beta \leqq$ $\nu(P) . \quad P$ is a copy of $Q$, and $\nu(F \cap P)=0$. By Lemma 2 there is a relatively open subset $G$ of $P$ such that $G \cap F \cap P=\varnothing$ and $\alpha<$ $\nu(G)<\beta$. Let $\pi$ denote the projection map of $Q$ onto $P$ and define $s_{1}(x)=d(\pi(x), P-G)$. For any $x=\left(x_{i}\right) \in Q$ and $0<\varepsilon<c$ define $g_{\mathrm{s}}(x)=\left(x_{\boldsymbol{i}}^{\prime}\right)$ by setting $x_{i}^{\prime}=x_{i}$ for all $i \neq k$ and letting $x_{k}^{\prime}$ be the 
piecewise linear function of $x_{k}$ that maps $0, c, 1$ into $0, c-\varepsilon s_{1}(x), 1$, respectively. Then $g_{\varepsilon} \in H_{0}(Q, B)$. Since $g_{\varepsilon}\left(R_{2}\right) \supset R_{2}$ we have $\nu g_{\varepsilon}\left(R_{2}\right) \geqq$ $\alpha_{2}$. Since $g_{\varepsilon}\left(R_{1}\right)$ increases to $R_{1}-G$ as $\varepsilon \downarrow 0$, and $\alpha_{1}<\nu\left(R_{1}-G\right)<$ $\alpha_{1}+1 / n$, we have $\alpha_{1}<\nu g_{\varepsilon}\left(R_{1}\right)<\alpha_{1}+1 / n$ for all sufficiently small $\varepsilon$, and then $g g_{\varepsilon} \in H-H_{n}$. This shows that $H_{n}$ is a nowhere dense subset of $H$. It follows that the equation $\mu h\left(R_{1}\right)=\alpha_{1}$ holds for all $h$ in a dense $G_{i}$ subset of $H$, and similarly the equation $\mu h\left(R_{2}\right)=\alpha_{2}$. Any $h$ belonging to the intersection of these two sets has the required properties.

By a simple partition $\mathscr{P}$ of $Q$ we shall mean a partition of $Q$ into $r$-sets defined by a finite number of sections of $Q$. Let $|\mathscr{P}|$ denote the maximum of the diameters of the members of $\mathscr{P}$.

LEMMA 4. Let 4 be a finite nonatomic Borel measure in $Q$ with $\mu(\delta Q)=0$, let $B$ be the union of a finite number of faces of $Q$, let $\left\{R_{1}, \cdots, R_{N}\right\}$ be a simple partition of $Q$, and let $\alpha_{1}, \cdots, \alpha_{N}$ be positive numbers with $\alpha_{1}+\cdots+\alpha_{N}=\mu(Q)$. Then there is an $h \in H_{0}(Q, B)$ such that $\mu h\left(R_{i}\right)=\alpha_{i}(i=1, \cdots, N)$.

Proof. This follows easily from Lemma 3 by induction on the number of sections, using the fact that each $r$-set is a copy of $Q$ and that its boundary is the union of a finite number of its faces.

Lemma 5. Let $\mu$ and $\nu$ be finite, nonatomic, locally positive Borel measures in $Q$ with $\mu(\delta Q)=\nu(\delta Q)=0$, let $B$ be the union of a finite number of faces of $Q$, and let $\mathscr{P}$ be a simple partition of $Q$ such that $\mu(R)=\nu(R)$ and $\mu(\delta R)=\nu(\delta R)=0$ for each $R \in \mathscr{P}$. For any $\varepsilon>0$ there exists a simple refinement $\mathscr{P}^{\prime}$ of $\mathscr{P}$ with $\left|\mathscr{P}^{\prime}\right|<$ $\varepsilon$, and an $h \in H_{0}(Q, B)$ that leaves each $R \in \mathscr{P}$ invariant and $\partial R$ fixed, such that $\nu\left(R^{\prime}\right)=\mu h\left(R^{\prime}\right)$ and $\nu\left(\delta R^{\prime}\right)=\mu h\left(\delta R^{\prime}\right)=0$ for each $R^{\prime} \in \mathscr{P}^{\prime}$.

Proof. Let $\mathscr{P}^{\prime}$ be a refinement of $\mathscr{P}^{\prime}$ with $\left|\mathscr{P}^{\prime}\right|<\varepsilon$ defined by taking additional sections of $Q$ that have $\nu$-measure zero. Apply

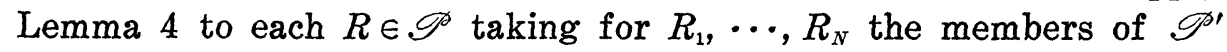
that are contained in $R$, with $\alpha_{i}=\nu\left(R_{i}\right)$ and $\partial R \cup(B \cap R)$ in place of $B$. The homeomorphisms so obtained fit together to define an $h \in H_{0}(Q, B)$ with the required properties.

Proof of Theorem 1. Assume that $\mu(\delta Q)=0$. For any $0<\varepsilon \leqq$ 1 and $h \in H(Q)$ let $\varphi(h, \varepsilon)$ be a positive number (necessarily $\leqq \varepsilon$ ) such that $\rho(h, h g)<\varepsilon$ whenever $g \in H(Q)$ and $\|g\|<\varphi(h, \varepsilon)$. By Lemma 5 there is a simple partition $\mathscr{P}_{1}$ of $Q$ with $\left|\mathscr{P}_{1}\right|<1$ and a $g_{1} \in$ $H_{0}(Q, B)$ such that $\mu(R)=\lambda g_{1}(R)$ and $\mu(\delta R)=\lambda g_{1}(\delta R)=0$ for $R \in \mathscr{P}_{1}$. 
Again by Lemma 5 there is a simple refinement $\mathscr{P}_{2}$ of $\mathscr{P}_{1}$ with $\left|\mathscr{P}_{2}\right|<\varphi\left(g_{1}, 1 / 2\right)$ and an $h_{1} \in H_{0}(Q, B)$ such that $\mu h_{1}(R)=\lambda g_{1}(R)$ and $\mu h_{1}(\delta R)=\lambda g_{1}(\delta R)=0$ for $R \in \mathscr{P}_{2}$. Applying Lemma 5 alternately to triples of the form $\mu h_{1} \cdots h_{n}, \lambda g_{1} \cdots g_{n}, \mathscr{P}_{2 n}$ and $\mu h_{1} \cdots h_{n}$, $\lambda g_{1} \cdots g_{n+1}, \mathscr{P}_{2 n+1}$ we can determine inductively a sequence $\left\{\mathscr{P}_{n}\right\}$ of simple partitions of $Q$, and sequences $\left\{g_{n}\right\}$ and $\left\{h_{n}\right\}$ in $H_{0}(Q, B)$ such that for each $n \geqq 1$ :

(i) $\mathscr{P}_{n+1}$ is a refinement of $\mathscr{P}_{n}$,

(ii) $\left|\mathscr{P}_{2 n}\right|<\varphi\left(g_{1} \cdots g_{n}, 1 / 2^{n}\right)$ and $\left|\mathscr{P}_{2 n+1}\right|<\varphi\left(h_{1} \cdots h_{n}, 1 / 2^{n}\right)$,

(iii) $g_{n+1}$ leaves each member of $\mathscr{P}_{2 n}$ invariant and $h_{n+1}$ leaves each member of $\mathscr{P}_{2 n+1}$ invariant,

(iv). $\mu h_{1} \cdots h_{n}(R)=\lambda g_{1} \cdots g_{n}(R)$ and $\mu h_{1} \cdots h_{n}(\delta R)=\lambda g_{1} \cdots g_{n}(\delta R)=$ 0 for $R \in \mathscr{P}_{2 n}$.

It follows from (ii) and (iii) that the limits $g=\lim g_{1} \cdots g_{n}$ and $h=\lim h_{1} \cdots h_{n}$ exist in $H_{0}(Q, B)$, and from (i), (iii) and (iv) that $\mu h(R)=\lambda g(R)$ and $\mu h(\delta R)=\lambda g(\delta R)=0$ for each $R \in \bigcup_{1}^{\infty} \mathscr{P}_{2 n}$. By (i) and (ii) each open set in $Q$ is the union of a sequence of nonoverlapping $r$-sets belonging to $\bigcup_{1}^{\infty} \mathscr{P}_{2 n}$. Hencè $\mu h(E)=\lambda g(E)$ for every open set $E$ and therefore for every Borel set in $Q$. Thus $f=g h^{-1}$ fulfills the requirements of Theorem 1 .

2. Refinements of Theorem 1. For some applications it is important to be able to effect the transformation of one measure into another by a homeomorphism that leaves fixed not only a finite number of faces of $Q$ but also some more general kinds of nowhere dense sets of measure zero. In this section we shall derive two such refinements of Theorem 1. First we need a preliminary result.

Lemma 6. Let $C$ be a closed subset of a section $P$ of $Q$, let $B$ be the union of a finite number of faces of $Q$, and let $\mu$ be a finite Borel measure in $Q$ with $\mu(B \cup C)=0$. There exists an arbitrarily small $h \in H_{0}(Q, B \cup C)$ such that $\mu h(P)=0$.

Proof. Let $\pi$ denote the projection map of $Q$ onto $P=\{x \in Q$ : $\left.x_{k}=c\right\}$. For any $x \in Q$ define $s(x)=d(\pi(x),(B \cap P) \cup C)$. (Put $s(x)=$ 1 in case $(B \cap P) \cup C=\varnothing$.$) For any x=\left(x_{i}\right) \in Q$ and $0<t<1-c$ define $h_{t}(x)=\left(x_{i}^{\prime}\right)$ by setting $x_{i}^{\prime}=x_{i}$ for $i \neq k$ and letting $x_{k}^{\prime}$ be the piecewise linear function of $x_{k}$ that maps $0, c, 1$ into $0, c+t s(x), 1$, respectively. Then $h_{t} \in H_{0}(Q, B \cup C)$. For $0<t<t^{\prime}<1-c$ the sets $h_{t}(P)$ and $h_{t^{\prime}}(P)$ are $\mu$-almost disjoint, since

$$
h_{t}(P) \cap h_{t^{\prime}}(P)=(B \cap P) \cup C .
$$

Hence $\mu h_{t}(P)>0$ for at most countably many values of $t$. Take $h=h_{t}$ for any sufficiently small $t$ that does not belong to this 
exceptional set.

LEMMA 7. Let $C$ be a closed subset of the intersection of two orthogonal sections $P$ and $P^{\prime}$ of $Q$, let $B$ be the union of a finite number of faces of $Q$, and let $\mu$ be a nonatomic, locally positive, normalized Borel measure in $Q$ with $\mu(\delta Q \cup C)=0$. Then $\mu=\lambda f$ for some $f \in H_{0}(Q, B \cup C)$.

Proof. By Lemma 6 there is an $h \in H_{0}(Q, B \cup C)$ such that $\mu h(P)=0$. If the conclusion holds for the measure $\mu h$, then it holds for $\mu$. Hence we may and shall assume henceforward that $\mu(P)=0$ and that $C=P \cap P^{\prime}$.

Let $R_{1}, R_{2}$ and $R_{1}^{\prime}, R_{2}^{\prime}$ be the $r$-sets into which $Q$ is divided by $P$ and $P^{\prime}$, respectively. Since $\mu(P)=0$ we have $\mu\left(\delta R_{1}\right)=\mu\left(\delta R_{2}\right)=0$, and since

$$
\mu\left(R_{i}\right) \lambda\left(R_{1}^{\prime}\right)+\mu\left(R_{i}\right) \lambda\left(R_{2}^{\prime}\right)=\mu\left(R_{i}\right)
$$

we can apply Lemma 3 to $R_{1}$ and $R_{2}$ separately and obtain an $h \in H_{0}(Q, B \cup P)$ such that

$$
\mu h\left(R_{\imath} \cap R_{j}^{\prime}\right)=\mu\left(R_{i}\right) \lambda\left(R_{j}^{\prime}\right) \quad(i, j=1,2) .
$$

Because these four numbers add up to 1 we have also $\mu h(P)=$ $\mu h\left(P^{\prime}\right)=0$. Summing over $i=1,2$ we get

$$
\mu h\left(R_{j}^{\prime}\right)=\lambda\left(R_{j}^{\prime}\right) \quad(j=1,2) .
$$

We can therefore apply Theorem 1 to the measures $\mu h$ and $\lambda$ in $R_{1}^{\prime}$ and $R_{2}^{\prime}$ separately (after normalizing them) and infer that $\mu h=\lambda g$ for some $g \in H_{0}\left(Q, B \cup P^{\prime}\right)$. Taking $f=g h^{-1}$ we have $\mu=\lambda f$ and $f \in H_{0}(Q, B \cup C)$.

Lemma 7 and the following theorem extend to $I^{\infty}$ the type of result embodied in Corollary 4 of [7] and Lemma 4 of [5], but the method of proof here is quite different.

THEOREM 2. Let $C$ be a closed subset of the intersection of two orthogonal sections $P$ and $P^{\prime}$ of $Q$, and let $B$ be the union of a finite number of faces of $Q$. If $\mu$ and $\nu$ are two nonatomic, locally positive Borel measures in $Q$ such that $\mu(Q)=\nu(Q)$ and

$$
\mu(B \cup C)=\nu(B \cup C)=0,
$$

then $\mu=\nu f$ for some $f \in H(Q, B \cup C)$.

Pooof. By Lemma 6 applied to $\mu+\nu$ there is a $g_{1} \in H_{0}(Q, B \cup C)$ such that $(\mu+\nu) g_{1}(P)=0$. We can apply Lemma 1 to each of the 
two $r$-sets into which $Q$ is divided by $P$ and obtain a $g_{2} \in H(Q, B \cup P)$ such that $(\mu+\nu) g_{1} g_{2}(\delta Q)=0$. Then $\mu g_{1} g_{2}(\delta Q \cup C)=\nu g_{1} g_{2}(\delta Q \cup C)=0$ and it follows from Lemma 7 that $\mu g_{1} g_{2}=\nu g_{1} g_{2} g_{3}$ for some $g_{3} \in$ $H_{0}(Q, B \cup C)$. Taking $f=g_{1} g_{2} g_{3} g_{2}^{-1} g_{1}^{-1}$ we have $\mu=\nu f$ and $f \in$ $H(Q, B \cup C)$.

A $Z$-set (in $Q$ ) is by definition a closed subset $A$ of $Q$ with the property that for each $\varepsilon>0$ there exists a continuous map $f: Q \rightarrow$ $Q-A$ such that $d(x, f(x))<\varepsilon$ for all $x \in Q$. It is known [2] that a closed set $A \subset Q$ is a $Z$-set if and only if it has topological infinite codimension; that is, for some $h \in H(Q)$ the image $h(A)$ projects to an interior singleton in infinitely many coordinates. Moreover, the following propositions are known (see [2]):

(2.1) If $A$ is a $Z$-set and $h \in H(Q)$, then $h(A)$ is a $Z$-set.

(2.2) Any compact subset of $s$ or of $\delta Q$ is a $Z$-set.

(2.3) Any finite union of $Z$-sets is a $Z$-set.

Any $Z$-set is closed and nowhere dense, but not conversely; for example, a section of $Q$ is not a $Z$-set, neither is the intersection of two sections, or a wild Cantor set [12]. The following theorem is therefore not as general as Theorem 2, but it is easier to apply since it does not require the fixed set to be in special position.

THEOREM 3. Let $\mu$ and $\nu$ be nonatomic, locally positive Borel measures in $Q$ with $\mu(Q)=\nu(Q)$, and let $A$ be a $Z$-set such that $\mu(A)=\nu(A)=0$. Then $\mu=\nu$ for some $f \in H(Q, A)$.

Proof. Let $h \in H(Q)$ be such that $h(A)$ projects into an interior singleton in infinitely many coordinates. In particular, $h(A)$ is contained in the intersection of two orthogonal sections of $Q$. Put $\mu^{\prime}=\mu h^{-1}$ and $\nu^{\prime}=\nu h^{-1}$. We can apply Theorem 2 to $\mu^{\prime}$ and $\nu^{\prime}$ with $C=h(A)$ and $B=\varnothing$ and infer that $\mu^{\prime}=\nu^{\prime} f$ for some $f \in H(Q, h(A))$. Then $\mu=\nu h^{-1} f h$ and $h^{-1} f h \in H(Q, A)$.

3. An extension theorem for measure preserving homeomorphisms. The following important Extension Theorem, due originally to R. D. Anderson, is known to hold [2]:

(3.1) If $A$ and $B$ are $Z$-sets in $Q$ and $h$ is a homeomorphism of $A$ ionto $B$ with $\sup _{x \in A} d(x, h(x))<\varepsilon$, then $h$ can be extended to a homeomorphism $\tilde{h} \in H(Q)$ with $\|\widetilde{h}\|<\varepsilon$.

We shall obtain from this a similar theorem for measure preserv- 
ing homeomorphisms except that the norm of $\tilde{h}$ can no longer be restricted.

THEOREM 4. Let $\mu$ be a measure in $Q$ homeomorphic to $\lambda$. If $A$ and $B$ are $Z$-sets in $Q$ and $h$ is a $\mu$-preserving homeomorphism of $A$ onto $B$, then $h$ can be extended to a u-preserving homeomorphism $\tilde{h} \in H(Q)$. However, there exist arbitrarily small $\mu$-preserving homeomorphisms $h$ for which the norm of any $\mu$-preserving extension $\tilde{h}$ must be arbitrarily close to 1.

Proof. By (3.1) $h$ can be extended to a homeomorphism $g \in$ $H(Q)$. Define Borel measures $\mu^{\prime}$ and $\nu^{\prime}$ in $Q$ by setting $\mu^{\prime}(E)=$ $\mu(E-A)$ and $\nu^{\prime}(E)=\mu g(E-A)$ for Borel sets $E$ in $Q$. Then $\mu^{\prime}$ and $\nu^{\prime}$ are nonatomic, locally positive (since $A$ is nowhere dense in $Q)$, and $\mu^{\prime}(Q)=\nu^{\prime}(Q)$. Evidently $\mu^{\prime}(A)=\nu^{\prime}(A)=0$. Hence $\mu^{\prime}=\nu^{\prime} f$ for some $f \in H(Q, A)$, by Theorem 3. Put $\tilde{h}=g f$. Then $\tilde{h}$ is $\mu$-preserving for subsets of $A$. For any Borel set $E \subset Q-A$ we have $E=E-A$ and $f(E)=f(E)-A$ and therefore

$$
\begin{aligned}
\mu \tilde{h}(E) & =\mu g f(E)=\mu g(f(E)-A)=\nu^{\prime}(f(E)) \\
& =\mu^{\prime}(E)=\mu(E-A)=\mu(E) .
\end{aligned}
$$

Thus $\tilde{h}$ is a $\mu$-preserving extension of $h$, as required.

That the norm of $\tilde{h}$ cannot be restricted in any way is shown by the following example, for which we are indebted to S. Alpern. Let $\varepsilon>0$ be given and suppose that $\mu=\lambda f$, where $f \in H(Q)$. There is a number $\eta>0$ such that $d\left(f^{-1}(x), f^{-1}(y)\right)<\varepsilon$ whenever $d(x, y)<$ $\eta$, and there exist basic open sets $U$ and $V$ such that $d\left(f^{-1}(x)\right.$, $\left.f^{-1}(y)\right)>1-\varepsilon$ for all $(x, y) \in U \times V . \quad U$ and $V$ are cylinder sets, say on $I^{n}$. Take a sufficiently fine dyadic subdivision of $I^{n}$ so that the $r$-sets $R_{1}, \cdots, R_{N}$ based on it have diameters less than $\eta$ and at least one is contained in $U$ and one in $V$. Let them be numbered so that $R_{1} \subset V, R_{K} \subset U$, and so that $R_{i}$ and $R_{i+1}$ have a face in common for $1 \leqq i<K$. For $1 \leqq i<K$ let $T_{i}$ denote the translation that maps $R_{i}$ onto $R_{i+1}$. Choose a $Z$-set $A_{1} \subset R_{1}-\delta R_{1}$ with ( $N$ 1) $\lambda\left(A_{1}\right)>(N-2) \lambda\left(R_{1}\right)$, and for $1<i \leqq N$ let $A_{i}$ be the image of $A_{1}$ by the translation that maps $R_{1}$ onto $R_{i}$. Put

$A=\cup\left\{A_{i}: 1 \leqq i \leqq N, \quad i \neq K\right\}$ and $B=\cup\left\{A_{i}: 1<i \leqq N\right\}$ and define $g: A \rightarrow B$ by setting $g=T_{i}$ on $A_{i}$ for $1 \leqq i<K$ and setting $g$ equal to the identity on $A_{i}$ sor $K<i \leqq N$. Then $A$ and $B$ are $Z$-sets, $g$ is a $\lambda$-preserving homeomorphism of $A$ onto $B$, and $d(x$, $g(x))<\eta$ for all $x \in A$. If $\widetilde{g}: Q \rightarrow Q$ is any invertible and $\lambda$-preserving extension of $g$ (not necessarily a homeomorphism!), then $\widetilde{g}$ maps $R_{K}$ onto a subset of $Q-B$. Since $R_{1} \subset Q-B$ and 


$$
\lambda\left(Q-B-R_{1}\right)=(N-1)\left(1 / N-\lambda\left(A_{1}\right)\right)<1 / N
$$

it follows that $\widetilde{g}\left(R_{K}\right)$ must meet $R_{1}$ in a set of positive measure. Put $h=f^{-1} g f$. Then $h$ is a $\mu$-preserving homeomorphism of the $Z$-set $f^{-1}(A)$ onto the $Z$-set $f^{-1}(B)$, and $d(x, h(x))<\varepsilon$ for all $x \in f^{-1}(A)$. If $\tilde{h} \in H(Q)$ is a $\mu$-preserving extension of $h$, then $f \widetilde{h} f^{-1}$ is a $\lambda$-preserving extension of $g$. Hence $f \widetilde{h} f^{-1}$ takes some point of $U$ into a point of $V$, and therefore $\|\tilde{h}\|>1-\varepsilon$.

4. Luzin's theorem for measure preserving homeomorphisms. Let $T$ be an invertible transformation of $I^{n}, n \geqq 2$, onto itself such that $T(E)$ and $T^{-1}(E)$ are measurable whenever $E$ is a measurable set. It was shown by Goffman [3] that there exists a homeomorphism $h$ of $I^{n}$ onto itself such that $h(x)=T(x)$ and $h^{-1}(x)=T^{-1}(x)$ except on a set of arbitrarily small Lebesgue measure. Goffman asked whether $h$ can be taken to be measure preserving in case $T$ is measure preserving. This was shown in [5] and independently by H. E. White, Jr. [11]. In this section we obtain corresponding theorems for $I^{\infty}$.

THEOREM 5. Let $\mu$ be a nonatomic, locally positive, normalized Borel measure in $Q$, let $B$ be the union of a finite number of faces of $Q$ with $\mu(B)=0$, and let $T$ be an invertible, Borel measurable and $\mu$-preserving transformation of $Q$ onto itself. For each $\varepsilon>0$ there exists a $\mu$-preserving homeomorphism $\tilde{h} \in H(Q, B)$ such that $\mu(\{x \in Q: \widetilde{h}(x) \neq T(x)\})<\varepsilon$.

Proof. We may assume without loss of generality that $\mu=\lambda$; because $\mu=\lambda f$ for some $f \in H(Q, B)$, by Theorem 1 , and it suffices to approximate the $\lambda$-preserving transformation $f T f^{-1}$ by a $\lambda$-preserving homeomorphism. By Luzin's theorem there exists a compact set $E$ with $\lambda(E)>1-\varepsilon$ such that the restriction of $T$ to $E$ is continuous and therefore a homeomorphism. Since $\lambda(\delta Q)=\lambda\left(T^{-1}(\delta Q)\right)=$ 0 we can choose a compact set

$$
C \subset E \cap s \cap T^{-1}(s) \text { with } \lambda(C)>1-\varepsilon .
$$

Let $h(x)=T(x)$ for $x \in C$, and $h(x)=x$ for $x \in B$. Then $h$ is a $\lambda$ preserving homeomorphism of $C \cup B$ onto $T(C) \cup B$, and both of these are $Z$-sets, by (2.2) and (2.3). The conclusion then follows from Theorem 4 .

An entirely different proof of Theorem 5 in the case $\mu=\lambda$ was found by S. Alpern and R. D. Edwards [1] shortly before Theorem 1 had been proved. They obtained $\tilde{h}$ by a limiting process that applies to $I^{n}$ for any $2 \leqq n \leqq \infty$ and involves neither homeomorphic 
measures nor extension of a homeomorphism from a subset. Their proof shows in addition that the norm of $\tilde{h}$ can be taken to be arbitrarily small provided $T$ belongs to a sufficiently small weak neighborhood of the identity. They observed also that this refinement of Theorem 5 is actually a corollary of that theorem; it suffices to divide $Q$ into $r$-sets $R_{i}$ of small diameter using sections of measure zero, to require that $T$ be weakly near enough to the identity so that it can be redefined on a set of small measure in such a way as to leave each $R_{i}$ invariant, and then to apply Theorem 5 to each of the sets $R_{i}$.

Goffman's theorem can be extended to $Q$ as follows (cf. [8]).

THEOREM 6. Let $T$ be an invertible and Borel measurable map of $Q$ onto itself, and let $\mu$ be a finite Borel measure in $Q$. For any $\varepsilon>0$ there is an $h \in H(Q)$ such that

$$
\mu\left(\left\{x \in Q: h(x) \neq T(x) \text { or } h^{-1}(x) \neq T^{-1}(x)\right\}\right)<\varepsilon .
$$

Proof. By Luzin's theorem there is a set $E$ with $\mu(E)>\mu(Q)-$ $\varepsilon / 2$ such that the restriction $T \mid E$ is continuous. The sets $s, T^{-1}(s)$ and their complements partition $E$ into four sets and we can choose compact subsets of each such that their union $K$ satisfies $\mu(K)>$ $\mu(Q)-\varepsilon / 2$. Then $T \mid K$ is a homeomorphism and both $K$ and $T(K)$ are $Z$-sets. Similarly we can find a compact set $K^{\prime}$ contained in $Q-T(K)$ with

$$
\mu\left(K^{\prime}\right)>\mu(Q-T(K))-\varepsilon / 2
$$

such that $T^{-1} \mid K^{\prime}$ is a homeomorphism and both $K^{\prime}$ and $T^{-1}\left(K^{\prime}\right)$ are $Z$-sets. Put $A=K \cup T^{-1}\left(K^{\prime}\right)$. This is a disjoint union of compact sets, so $T \mid A$ is a homeomorphism of $A$ onto the set $B=T(K) \cup K^{\prime}$, and both $A$ and $B$ are $Z$-sets. By (3.1) there is an $h \in H(Q)$ that agrees with $T$ on $A$. The conclusion then follows from the fact that the set where $h$ differs from $T$, or $h^{-1}$ from $T^{-1}$, is contained in $(Q-A) \cup(Q-B)$, which has $\mu$-measure less than $\varepsilon$.

REMARK. In case $\mu$ is nonatomic and locally positive, the proof of Theorem 4 (with $\mu^{\prime}$ and $\nu^{\prime}$ normalized) shows that the extension of $T \mid A$ can be made to be relatively $\mu$-preserving on $Q-A$. Consequently, in case $\mu$ is nonatomic and locally positive and $T$ preserves $\mu$-nullsets the $h$ in Theorem 6 can likewise be taken to preserve $\mu$-nullsets, and thus be "absolutely measurable" (cf. White [11]).

5. Sets topologically equivalent to nullsets. The following 
theorem extends Theorem 9 of [7] to $I^{\infty}$ and at the same time makes its formulation more complete. The completion of a Borel measure $\mu$ will be denoted by $\bar{\mu}$.

THEOREM 7. The following statements about a set $E \subset Q$ are pairwise equivalent:

(1) For each $\sigma$-finite Borel measure $\mu$ in $Q$ and $Z$-set $B$ with $\mu(B)=0$ there is an arbitrarily small $h \in H(Q, B)$ such that $\bar{\mu}(h(E))=0$.

(2) $\bar{\lambda}(h(E))=0$ for some $h \in H(Q)$.

(3) $Q-E$ contains a sequence of perfect sets whose union is dense in $Q$.

Proof. The implications $(1) \Rightarrow(2) \Rightarrow(3)$ are clear. In case $\mu$ is nonatomic the implication $(3) \Rightarrow(1)$ can be deduced from Theorem 3 by the method used in [7], but this method will not serve in general. Instead we base our proof on the following lemma.

LemmA 8. Given $\varepsilon>0$ and disjoint $Z$-sets $A$ and $C$ in $Q$, where $C$ is a Cantor set, (3) implies that there exists a Cantor $Z$ set $\Gamma$ contained in $Q-E-A$ and an $h \in H(Q, A)$ such that $h(\Gamma)=$ $C$ and $\|h\|<\varepsilon$.

Proof. Cover $C$ by disjoint open sets $U_{1}, \cdots, U_{n}$ that have diameters less than $\varepsilon$ and meet $C$ but not $A$. Each $U_{i}$ meets some perfect set contained in $Q-E$, hence we can find Cantor sets $\Gamma_{1}, \cdots, \Gamma_{n}$, each contained either in $s$ or $\delta Q$, such that $\Gamma_{i} \subset Q-E \cap$ $U_{i}(i=1, \cdots, n)$. Put $\Gamma=\bigcup_{1}^{n} \Gamma_{i}$ and let $\bar{h}: A \cup \Gamma \rightarrow A \cup C$ be a homeomorphism equal to the identity on $A$ and such that $\bar{h}\left(\Gamma_{i}\right)=C \cap$ $U_{i}(i=1, \cdots, n)$. By (3.1) $\bar{h}$ can be extended to a homeomorphism $h: Q \rightarrow Q$ with $\|h\|<\varepsilon$, and we have $h(\Gamma)=C$ and $h \in H(Q, A)$ as required.

$(3) \Rightarrow(1)$. Choose a sequence of mutually disjoint Cantor $Z$-sets $C_{j} \subset Q-B$ such that

$$
\mu\left(Q-\bigcup_{1}^{\infty} C_{j}\right)=0 .
$$

By Lemma 8 with $A=B, C=C_{1}$, and any $\varepsilon_{0}>0$, there exists a Cantor $Z$-set $\Gamma_{1} \subset Q-E-B$ and an $h_{1} \in H(Q, B)$ with $\left\|h_{1}\right\|<\varepsilon_{0} / 2$ such that $h_{1}\left(\Gamma_{1}\right)=C_{1}$. Suppose disjoint Cantor $Z$-sets $\Gamma_{1}, \cdots, \Gamma_{n}$ contained in $Q-E-B$ and $h_{1}, \cdots, h_{n}$ in $H(Q, B)$ have been defined so that for $j=1, \cdots, n$,

(i) $h_{j}\left(\Gamma_{j}\right)=C_{j}$,

(ii) $h_{j-1}^{-1} h_{j}$ is equal to the identity on $B \cup \cup_{1}^{j-1} \Gamma_{k}$, 
and

(iii) $\rho\left(h_{j-1}, h_{j}\right)<\varepsilon_{0} / 2^{j}$,

where $h_{0}=\mathrm{id}_{Q}$. Choose $\varepsilon_{n}>0$ so that

$$
\rho\left(h_{n}, h_{n} g\right)<\varepsilon_{0} / 2^{n+1}
$$

whenever $\|g\|<\varepsilon_{n}$. By Lemma 8 with $B \cup \bigcup_{1}^{n} \Gamma_{j}, h_{n}^{-1}\left(C_{n+1}\right), \varepsilon_{n}$ in place of $A, C, \varepsilon$ there is a Cantor $Z$-set

$$
\Gamma_{n+1} \subset Q-E-B-\bigcup_{1}^{n} \Gamma_{j}
$$

and a $g \in H\left(Q, B \cup \bigcup_{1}^{n} \Gamma_{j}\right)$ such that $g\left(\Gamma_{n+1}\right)=h_{n}^{-1}\left(C_{n+1}\right)$ and $\|g\|<\varepsilon_{n}$. Putting $h_{n+1}=h_{n} g$ it follows that (i) (ii) (iii) hold for $j=1, \cdots, n+1$. Thus sequences $\left\{\Gamma_{j}\right\}$ and $\left\{h_{j}\right\}$ can be defined inductively so that (i) (ii) (iii) are satisfied for all $j \geqq 1$. Then $\left\{h_{j}\right\}$ converges uniformly to a homeomorphism $h \in H(Q, B)$ with $\|h\|<\varepsilon_{0}$ such that $h\left(\Gamma_{j}\right)=C_{j}$ for all $j \geqq 1$. Since

$$
E \subset Q-\bigcup_{1}^{\infty} \Gamma_{j}
$$

we have $h(E) \subset Q-\bigcup_{1}^{\infty} C_{j}$ and therefore $\bar{\mu}(h(E))=0$.

The following corollary is a far-reaching generalization of Lemma 1.

THEOREM 8. If $\mu$ is a $\sigma$-finite Borel measure in $Q, B$ is the union of a finite number of faces of $Q$ with $\mu(B)=0$, and $A$ is an $F_{\sigma}$ set of first category in $Q$, then $\mu h(A)=0$ for all $h$ in a certain dense $G_{\dot{o}}$ subset of $H(Q, B)$.

Proof. For any nowhere dense closed set $F$ in $Q$ and any $g$ in $H(Q, B)$ we can take $E=g(F)$ in (3) of Theorem 7. Then (1) implies that for each positive integer $j$ the set

$$
E_{j}=\{h \in H(Q, B): \mu h(F)<1 / j\}
$$

is dense in $H(Q, B)$. Since $E_{j}$ is obviously open the conclusion follows.

The corresponding result for Lebesgue measure in $I^{n}$ ([6], Theorem 1) can be shown to hold also for the completion of any $\sigma$-finite Borel measure that vanishes on $\partial I^{n}$.

In concluding this section we remark that the proofs of Theorems 8 and 10 of [7] carry over to $I^{\infty}$ without change.

6. Measure preserving embeddings in metric measure spaces. By a metric measure space $(X, \bar{\mu})$ we mean in this section a metric space $X$ together with the completion $\bar{\mu}$ of a finite Borel measure 
$\mu$ in $X$. Let $\mu^{*}$ denote the outer measure in $X$ induced by $\mu$ (so that $\bar{\mu}$ is just $\mu^{*}$ restricted to the $\mu^{*}$-measurable sets). $\left(X_{1}, \bar{\mu}_{1}\right)$ is called a subspace of $(X, \bar{\mu})$ if $X_{1} \subset X$ and $\mu_{1}^{*}$ is the restriction of $\mu^{*}$ to subsets of $X_{1} . \quad(X, \bar{\mu})$ is said to be homeomorphic to $(Y, \bar{\nu})$ if there exists a homeomorphism of $X$ onto $Y$ that makes $\bar{\nu}$ correspond to $\bar{\mu}$. Such a homeomorphism of $(X, \bar{\mu})$ onto a subspace of $(Y, \bar{\nu})$ is called an embedding of $(X, \bar{\mu})$ in $(Y, \bar{\nu})$. In [9] and [10] A. $H$. Stone considered the question: What metric measure spaces ( $X$, $\bar{\mu})$ can be embedded in $(Q, \bar{\lambda})$ ? Obviously $X$ must be separable, and $\mu$ must be nonatomic with $\mu(X) \leqq 1$. Assuming $\mu(X)<1$, Stone obtained such an embedding in case $X$ is finite-dimensional. Using Theorem 1 in somewhat the same way as Stone used Theorem 2 of [7] we obtain the following answer to his question.

THEOREM 9. Let $X$ be a separable metric space and let $\bar{\mu}$ be the completion of a nonatomic Borel measure $\mu$ in $X$. There exists an embedding of $(X, \bar{\mu})$ in $(Q, \bar{\lambda})$ if and only if either $(1) \mu(X)<1$, or $(2) \mu(X)=1, \mu$ is locally positive, and $X$ is homeomorphic to some dense subset of $Q$.

Proof. The necessity of either (1) or (2) is clear. Assume first that $\mu(X)<1$ and let $f: X \rightarrow Y$ be a homeomorphism of $X$ onto a subset $Y$ of some section of $Q$, so that $\bar{\lambda}(Y)=0$. For each Borel set $E$ in $Q$ define.

$$
\nu(E)=\mu\left(f^{-1}(E \cap Y)\right)+(1-\mu(X)) \lambda(E) .
$$

Then $\nu$ is a nonatomic, locally positive, normalized Borel measure in $Q$. By Theorem $1, \nu=\lambda g$ for some $g \in H(Q)$, and so $\nu^{*}=\lambda^{*} g$. Then $h=g \circ f$ is a homeomorphism of $X$ onto $g(Y)$, and for any set $A \subset X$ we have

$$
\begin{aligned}
\lambda^{*} h(A) & =\nu^{*} f(A)=\mu^{*} f^{-1}(f(A) \cap Y)+(1-\mu(X)) \lambda^{*} f(A) \\
& =\mu^{*}(A \cap X)+0=\mu^{*}(A) .
\end{aligned}
$$

Thus $h$ is an embedding of $(X, \bar{\mu})$ in $(Q, \bar{\lambda})$.

Assuming (2), let $f$ be a homeomorphism of $X$ onto a dense subset $Y$ of $Q$, and define $\nu(E)=\mu f^{-1}(E \cap Y)$ for Borel sets $E$ in $Q$. By Theorem $1, \nu=\lambda g$ for some $g \in H(Q)$. Putting $h=g \circ f$ it follows, as above, that $\lambda^{*} h(A)=\mu^{*}(A)$ for every set $A \subset X$.

Theorem 9 leaves open the question of what spaces can be densely embedded in $Q$, but for compact spaces it furnishes a complete answer to Stone's question: $A$ compact metric measure space $(X, \bar{\mu})$ can be embedded in $(Q, \bar{\lambda})$ if and only if either $(X, \bar{\mu})$ is homeomorphic to $(Q, \bar{\lambda})$, or $\mu(X)<1$ and $\mu$ is nonatomic. 
Note added in proof. In "A note on topological measure theory" (preprint) Bejamin Weiss has independently obtained a different proof of Theorem 1 in the case $B=\varnothing$ and used it as above to answer Stone's question for compact metric measure spaces.

\section{REFERENCES}

1. S. Alpern and R. D. Edwards, Lusin's theorem for measure preserving homeomorphisms, (to appear).

2. T. A. Chapman, Lectures on Hilbert cube manifolds, C.B.M.S. Regional conference series in mathematics No. 28, Amer. Math. Soc., Providence, R.I., 1977.

3. C. Goffman, One-one measurable transformations, Acta Math., 89 (1953), 261-278.

4. C. Goffman and G. Pedrick, A proof of the homeomorphism of Lebesgue-Stieltjes measure with Lebesgue measure, Proc. Amer. Math. Soc., 52 (1975), 196-198.

5. J. C. Oxtoby, Approximation by measure-preserving homeomorphisms, Recent Advances in Topological Dynamics; Lecture Notes in Mathematics Vol. 318, Springer, Berlin, 1973, 206-217.

6. J. C. Oxtoby and S. M. Ulam, On the equivalence of any set of first category to a set of measure zero, Fund. Math., 31 (1938), 201-206.

7. - Measure-preserving homeomorphisms and metrical transitivity, Ann. of Math., (2) 42 (1941), 874-920.

8. V. S. Prasad, Approximation of 1-1 measurable transformations of $I^{\infty}$ by homeomorphisms, Notices Amer. Math. Soc., 23 (1976), A-485.

9. A. H. Stone, Topology and measure theory, Measure Theory, Oberwolfach 1975; Lecture Notes in Mathematics Vol. 541, Springer, Berlin, 1976, 43-48.

10. - Measure-preserving maps, Prague Symposium on General Topology, August 1976 .

11. H. E. White Jr., The approximation of one-one measurable transformations by measure-preserving homeomorphisms, Proc. Amer. Math. Soc., 44 (1974), 391-394.

12. R. Y. T. Wong, A wild Cantor set in the Hilbert cube, Pacific J. Math., 24 (1968), 189-193.

Received October 3, 1977. This paper includes results from the second author's doctoral dissertation (Bryn Mawr College, 1977). He acknowledges partial support from the National Research Council of Canada.

BRYN MAWR COLLEge

BRYN MAWR, PA 19010

AND

MCGILL UNIVERSITY

Montreal, Quebec, Canada 



\title{
PACIFIC JOURNAL OF MATHEMATICS
}

\section{EDITORS}

RICHARD ARENS (Managing Editor)

University of California

Los Angeles, CA 90024

Charles W. Curtis

University of Oregon

Eugene, OR 97403

C. C. MOORE

University of California

Berkeley, CA 94720
J. DUGUNDJI

Department of Mathematics

University of Southern California

Los Angeles, CA 90007

R. Finn and J. Milgram

Stanford University

Stanford, CA 94305

\section{ASSOCIATE EDITORS}
E. F. BECKENBACH
B. H. Neumann
F. WOLF
K. YosHidA

\section{SUPPORTING INSTITUTIONS}

\author{
UNIVERSITY OF BRITISH COLUMBIA \\ CALIFORNIA INSTITUTE OF TECHNOLOGY \\ UNIVERSITY OF CALIFORNIA \\ MONTANA STATE UNIVERSITY \\ UNIVERSITY OF NEVADA, RENO \\ NEW MEXICO STATE UNIVERSITY \\ OREGON STATE UNIVERSITY \\ UNIVERSITY OF OREGON
}

\author{
UNIVERSITY OF SOUTHERN CALIFORNIA \\ STANFORD UNIVERSITY \\ UNIVERSITY OF HAWAII \\ UNIVERSITY OF TOKYO \\ UNIVERSITY OF UTAH \\ WASHINGTON STATE UNIVERSITY \\ UNIVERSITY OF WASHINGTON
}

The Supporting Institutions listed above contribute to the cost of publication of this Journal, but they are not owners or publishers and have no responsibility for its content or policies.

Mathematical papers intended for publication in the Pacific Journal of Mathematics should be in typed form or offset-reproduced, (not dittoed), double spaced with large margins. Please do not use built up fractions in the text of the manuscript. However, you may use them in the displayed equations. Underline Greek letters in red, German in green, and script in blue. The first paragraph or two must be capable of being used separately as a synopsis of the entire paper. Items of the bibliography should not be cited there unless absolutely necessary, in which case they must be identified by author and journal, rather than by item number. Manuscripts, in triplicate, may be sent to any one of the editors. Please classify according to the scheme of Math. Reviews, Index to Vol. 39. All other communications should be addressed to the managing editor, or Elaine Barth, University of California, Los Angeles, California, 90024.

50 reprints to each author are provided free for each article, only if page charges have been substantially paid. Additional copies may be obtained at cost in multiples of 50 .

The Pacific Journal of Mathematics is issued monthly as of January 1966. Regular subscription rate: $\$ 72.00$ a year (6 Vols., 12 issues). Special rate: $\$ 36.00$ a year to individual members of supporting institutions.

Subscriptions, orders for numbers issued in the last three calendar years, and changes of address should be sent to Pacific Journal of Mathematics, P.O. Box 969, Carmel Valley, CA 93924, U.S.A. Older back numbers obtainable from Kraus Periodicals Co., Route 100, Millwood, NY 10546.

PUBLISHED BY PACIFIC JOURNAL OF MATHEMATICS, A NON-PROFIT CORPORATION

Printed at Kokusai Bunken Insatsusha (International Academic Printing Co., Ltd.). 8-8, 3-chome, Takadanobaba, Shinjuku-ku, Tokyo 160, Japan.

Copyright (C) 1978 by Pacific Journal of Mathematics Manufactured and first issued in Japan 


\section{Pacific Journal of Mathematics}

Vol. 77, No. $2 \quad$ February, 1978

Graham Donald Allen, Duals of Lorentz spaces ................... 287

Gert Einar Torsten Almkvist, The number of nonfree components in the decomposition of symmetric powers in characteristic p .......... 293

John J. Buoni and Bhushan L. Wadhwa, On joint numerical ranges ...... 303

Joseph Eugene Collison, Central moments for arithmetic functions . . . . . . 307

Michael Walter Davis, Smooth G-manifolds as collections of fiber

bundles ........................................ 315

Michael E. Detlefsen, Symmetric sublattices of a Noether lattice......... 365

David Downing, Surjectivity results for $\phi$-accretive set-valued

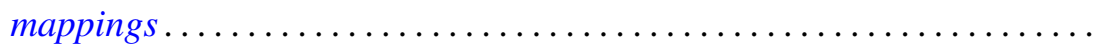

David Allyn Drake and Dieter Jungnickel, Klingenberg structures and partial designs. II. Regularity and uniformity ................. 389

Edward George Effros and Jonathan Rosenberg, $C^{*}$-algebras with approximately inner flip .......................... 417

Burton I. Fein, Minimal splitting fields for group representations. II. . . . . 445

Benjamin Rigler Halpern, A general coincidence theory ............. 451

Masamitsu Mori, A vanishing theorem for the mod $p$ Massey-Peterson spectral sequence ................................ 473

John C. Oxtoby and Vidhu S. Prasad, Homeomorphic measures in the

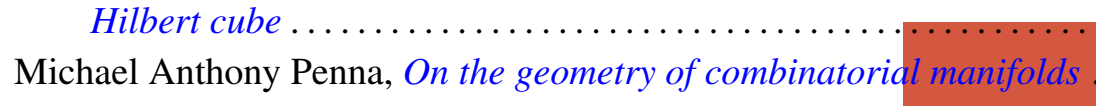

Robert Ralph Phelps, Gaussian null sets and differentiability of Lipschitz map on Banach spaces........................

Herbert Silverman, Evelyn Marie Silvia and D. N. Telage, Locally univalent functions and coefficient distortions...

Donald Curtis Taylor, The strong bidual of $\Gamma(K)$

Willie Taylor, On the oscillatory and asymptotic behavior of solutions of fifth order selfadjoint differential equations ...........

Fu-Chien Tzung, Sufficient conditions for the set of Hausdorff compactifications to be a lattice. 